\title{
Numerical model of a solar ventilated facade element: experimental validation, final parameters and results
}

\author{
Andrei-Stelian Bejan ${ }^{1,2^{*}}$, Florin Bode ${ }^{2,3}$, Cătalin Teodosiu ${ }^{1,2}$, Cristiana Verona Croitoru ${ }^{1,2}$, and Ilinca Năstase $^{1,2}$ \\ ${ }^{1}$ Technical University of Civil Engineering Bucharest, Faculty of Engineering for Building Services, 021407 Bucharest, Romania \\ ${ }^{2}$ CAMBI Research Center, 021407 Bucharest, Romania \\ ${ }^{3}$ Technical University of Cluj-Napoca, 400114 Cluj-Napoca, Romania
}

\begin{abstract}
The present paper analyses the airflow through the lobed orifices of a transpired solar collector which acts as a solar ventilated facade element through numerical simulation. This study is part of a complex research project which analyses the implementation of phase changing materials within air solar collectors. We decided to study an elementary part of the collectors' absorbent plate with four equivalent orifices in order to obtain the velocity and temperature field at the outlet of the computing domain since the numerical simulation of the entire solar collector (more than 5000 orifices) is not feasible due to the big amount of computational resources and time needed. This paper presents the experimental validation of the numerical model, its final parameters and preliminary results. The numerical simulation was conducted using Ansys Fluent CFD software and the results were processed via Tecplot. The boundary conditions imposed were emphasised and k- $\varepsilon$ RNG turbulence model was used according to the literature. After comparing the velocity profiles and temperature fields obtained with both experimental and numerical approaches we concluded that the numerical model reproduces real flow phenomena within acceptable limits. The numerical model thus obtained will be used in further studies in order to optimise the collectors' geometry and characteristics by means of parametrical analyses.
\end{abstract}

\section{Introduction}

\subsection{Objective}

The present paper is part of a complex research conducted by our team which analyses the implementation of phase changing materials (PCMs) within transpired air solar collectors (TSCs) with lobed orifices acting as a solar ventilated facade element.

During the construction of the TSC numerical model we understood that the numerical simulation of the entire solar collector absorber metal plate $(1020 \times 2000 \mathrm{~mm}$ and 5000 orifices) is not feasible thus resulting a huge number of cells for the numerical mesh for which we would have needed very high computational resources and a very large amount of time in order to solve the problem.

Taking these aspects into consideration we decided to create first a numerical model that can accurately simulate the airflow through the lobed orifices. The results obtained at the outlet of the current model will be used as boundary conditions at the inlet of the actual solar collector numerical model which will be presented in further studies.

The aim of the present paper is to present the final parameters and results of the numerical model that studies the airflow through the lobed orifices of the TSC and analyses the experimental validation of the model that has been conducted after the mesh independency study presented in previous studies [1].

Regardless of their type, the operating principle of air solar collectors is the same and it is very simple [2]. Solar radiation is captured by an absorbent element (generally a metal plate) that transfers the heat to the airflow within the collectors' air cavity.

Regarding the transpired collectors, the energy is captured by a metal plate with perforations and then it is ceded to the air which is collected at the top of the collector in a mixing box and introduced into the room by using a ventilator $[2,3]$ (during the winter or during transition periods). If there are thermal energy storage materials integrated, the energy stored during the day when the solar radiation is available could be later ceded at night thus increasing the global efficiency of air solar collector and its number of operating hours $[4,5]$.

Transpired solar collectors (TSCs) typically have a low cost of investment and a good efficiency in the case of space heating, fresh air preheating or drying systems [6] and they have low operational costs [7]. Transpired solar collectors are typically used for applications where the required temperature is not very high [8] and the side winds are not very powerful [9].

Van Decker et al. [10] highlights in his researches that more than $28 \%$ of the thermal energy is ceded to the air in the perforations. Many of studies regarding the airflow through different perforations have been

* Corresponding author: andrei.s.bejan@gmail.com 
conducted lately [11-14]. We used lobed shaped orifices for the study taken in this article, because according to the literature $[2,3]$, the lobed geometries (unlike the classical circular ones) are $15 \%$ more efficient for flows ranging from $80 \mathrm{~m}^{3} / \mathrm{h} / \mathrm{m}^{2}$ to $220 \mathrm{~m}^{3} / \mathrm{h} / \mathrm{m}^{2}$ and can determine an outlet air temperature higher with up to $2{ }^{\circ} \mathrm{C}$. The unconventional shape of the lobed orifices determines a more complex flow that improves heat transfer by approximately $40 \%$.

In order to optimize the geometry and parameters of a TSC it is necessary to run numerical studies. According to the literature one of the software often used to simulate the TSCs is Ansys Fluent CFD software [1517].

The present paper presents the methodology used for the numerical studies conducted and highlights a synthesis of the collector geometry and mesh independency study conducted by our team [1]. The experimental validation of the numerical study was made by taking into account two different approaches: by comparing the velocity fields in two longitudinal planes with PIV experimental results and by comparing the temperatures obtained in the outlet of the computing domain with experimental results. The final parameters and preliminary results of the numerical model are presented also in the final chapter.

\subsection{Methodology}

Computational Fluid Dynamics (CFDs) is a widely used numerical modelling technique in the engineering and research. CFDs technique is based on the fundamental equations governing fluid dynamics: the continuity equation, the energy conservation equation and the impulse conservation equation. Because the results of Navier-Stokes equations cannot be solved analytically, the CFD software tries to find an approximate solution by spatial meshing methods that convert partialderivative equations into algebraic equations (calculations are iterative).

The stages of a computational fluid dynamics simulation that were conducted for the present study are: the construction of the numerical model geometry, the mesh independency study, imposing the settings of the numerical case (boundary conditions, turbulence model etc.), experimental validation of the numerical model and calculation of the solution (parametrical studies, optimisation and post-processing).

Previous studies conducted by our research team covers the first three steps mentioned above [1], while the present paper analyses the last two stages of the numerical study. Parametrical studies (post-processing) will be performed in order to optimize the solar collector geometry and characteristics. Moreover, further studies will be conducted in order to assess the implementation of PCMs in TSCs.

Numerical simulation software CFD ANSYS Fluent [18] and all its components: ANSYS DesignModeler, ANSYS Workbench and ANSYS Mesher were used to carry out the numerical study and Tecplot software was used for data processing. Moreover, one computing station (Intel Xeon $3.4 \mathrm{GHz}, 16$ cores, 128 GB RAM) which is in the endowment of Technical University of Civil Engineering laboratories was used for the numerical simulations.

\section{Geometry and method}

\subsection{Geometry and functioning}

The geometry of the solar collector is widely presented in literature $[1,19]$ and it is synthesized below (figure 1 and 2) in order to better understand the present study.

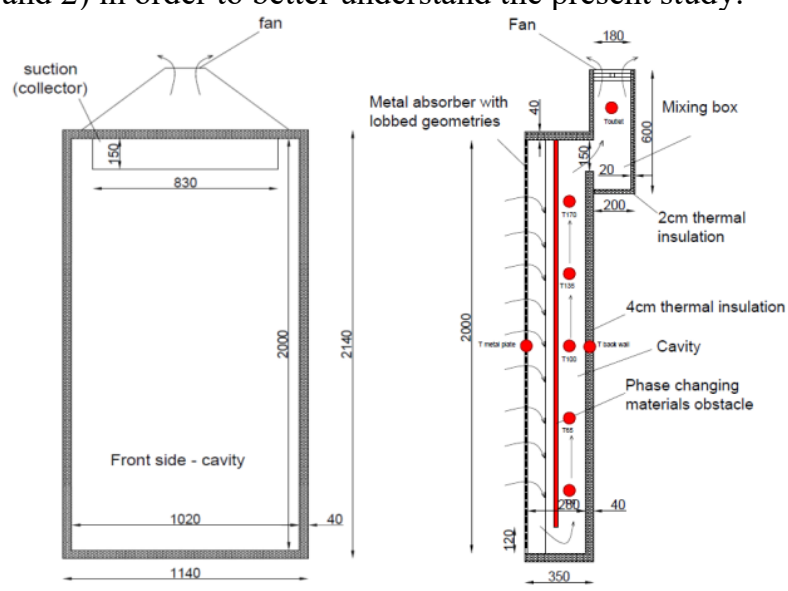

Fig. 1. Geometry of the actual transpired solar collector with integrate phase changing materials which is the subject of the present research [19].

The ambient air enters the TSCs cavity through the lobed orifices of the metal absorber while being preheated, is forced to flow to the bottom of the collector by the PCM obstacle, then enters the 2 nd air cavity and takes an upward trajectory being exhausted through the outlet using a fan.

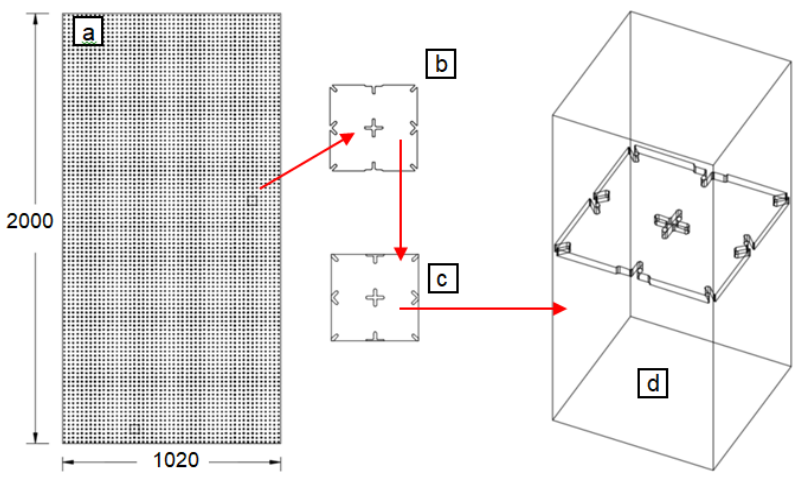

Fig. 2. Designing the 3D model in AutoCAD: a - absorbent metal plate with lobed orifices, $b$ - extraction of a $4 \times 4 \mathrm{~cm}$ part, $\mathrm{c}-$ embedment in the computing domain, $\mathrm{d}-3 \mathrm{D}$ computing domain $(4 \times 4 \mathrm{~cm}$ plate surrounded by the computational domain) [1].

In order to be able to perform the numerical modelling of the TSCs with lobed perforations and integrated PCMs in a feasible period of time, we have simplified as much as possible the geometry of the absorber plate that is part of the solar collector, starting 
from the premise of the thermal transfer phenomena symmetry and velocity fields symmetry (figure 2).

For the present study only, the numerical model of the air flow through the lobed orifices was realised in order to assess the impact of the orifices. For the further studies the results of this model will be transposed as boundary conditions for the actual solar collector in order to assess the impact of PCMs integration.

The absorbent metal plate is 1020 x $2000 \mathrm{~mm}$ and presents 5000 lobed orifices. Considering the airflow and heat transfer phenomena symmetry we selected a $4 \times 4 \mathrm{~cm}$ metal plate with 4 equivalent orifices for the present study (figure 2b). The absorbent plate is positioned at $4 \mathrm{~cm}$ distance from the computing domain limit (inlet upstream) and at $5 \mathrm{~cm}$ distance from the outlet (downstream) which represents ten equivalent diameters $(10 \mathrm{De}=5 \mathrm{~cm})$. The 3D geometry was drawn in AutoCAD and imported in ANSYS DesignModeler (figure 3). This computing domain was chosen because we observed from the experimental studies that after $5 \mathrm{~cm}$ the velocity fields are rapidly becoming uniform.

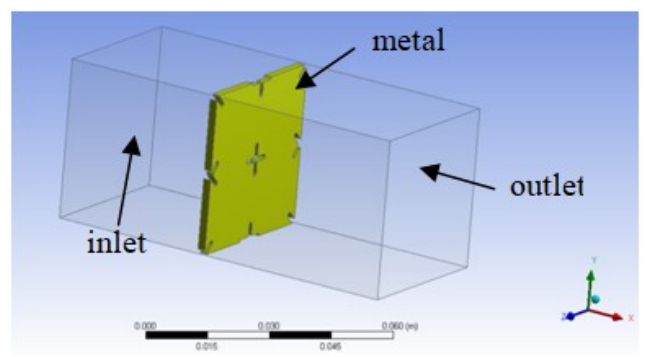

Fig. 3. Importing the geometry from AutoCAD to ANSYS DesignModeler - isometric view [1].

\subsection{Meshing}

In order to determine the number of computing elements (cells) useful to solve the problem, we conduct a solution independency study based on the quality of the mesh or mesh independency study which was presented in previous studies [1]. After comparing the velocity and temperature fields obtained within experimental and numerical studies (in longitudinal and transverse planes) we conclude that the geometry of 5.3 million cells is an independent solution to the computing grid and this will be used in the numerical model analysed (figure 4).
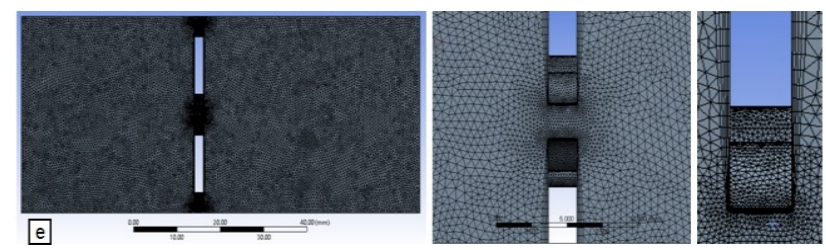

Fig. 4. 5.3 million elements mesh level chosen after the mesh independency study conducted [1].

For the mesh independency study the following boundary conditions were used: average velocity at the computing domain limit - inlet $(0.0555 \mathrm{~m} / \mathrm{s})$, inlet temperature $\left(25^{\circ} \mathrm{C}\right)$ and metal plate temperature $\left(50{ }^{\circ} \mathrm{C}\right)$.
The models used for the numerical case were the energy model which actives the energy equation and the k- $\varepsilon$ RNG turbulence model (with Enhanced Wall Function EWF) which is suitable, according to the literature studied [20], in the cases with swirling flows and in the case of flows with small Reynolds numbers ( $R e \approx 422$ in the present study).

\section{Experimental validation of the numerical model}

The mesh independency study must be followed by the experimental validation of the numerical model in order to achieve the final numerical model. This will be achieved through two different approaches:

- By comparing the air velocity profiles in longitudinal planes (numerical results vs. PIV experimental results)

- By comparing the temperatures resulted on the computing domain outlet (numerical vs. experimental).

\subsection{Velocity profiles comparison in longitudinal plane}

In order to validate the velocity profiles in longitudinal planes, measurements were made using PIV method (Particle Image Velocimetry or Particle Image Speed Measurement) and compared with the results of numerical simulations obtained under similar conditions.
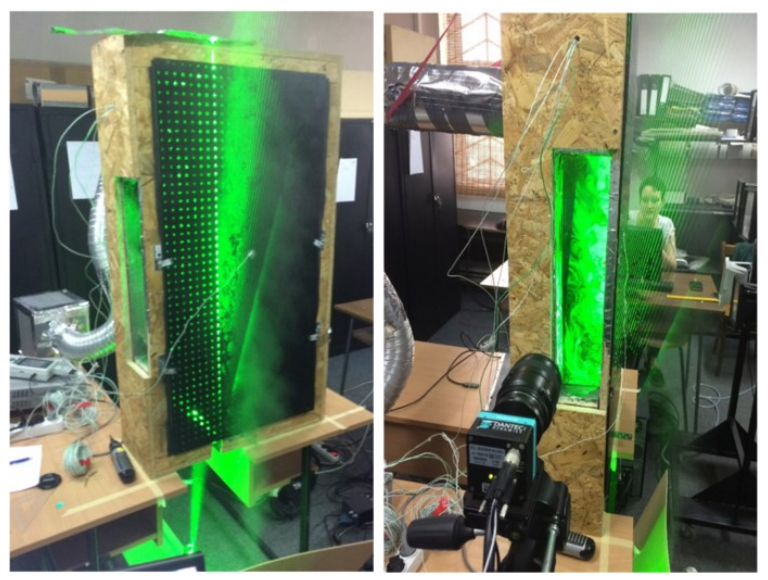

Fig. 5. The use of the PIV technique in order to measure air velocity profiles as a results of a metal absorber with lobed orifices - small size collector (according to [21]).

The PIV technique is a method of studying the flows generated by various grilles/air diffusion units that allows the measurement of instantaneous velocity fields (figure 5). The experimental results used to validate the numerical model were taken from previous studies conducted within the Faculty of Building Services Engineering (Technical University of Civil Engineering, Bucharest) [2], studies that pursued the analysis of the velocity profiles for a water flow which passes through similar lobed orifices in case of a certain Reynolds number: $\mathrm{Re}=2500$.

For the experimental validation of the numerical model, we analysed the XY longitudinal planes, in the 
plane that traverses the centre of the lobed orifice (the centre of the jet $-Z=0 \mathrm{~mm}$ ) and at $2.5 \mathrm{~mm}$ distance $(Z=2.5 \mathrm{~mm})$, as can be seen in figure 6 .

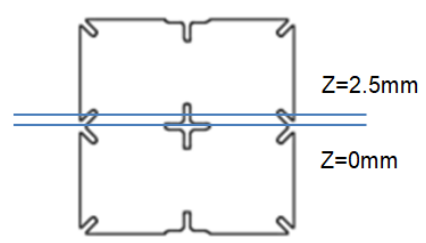

Fig. 6. Longitudinal planes used for experimental validation.

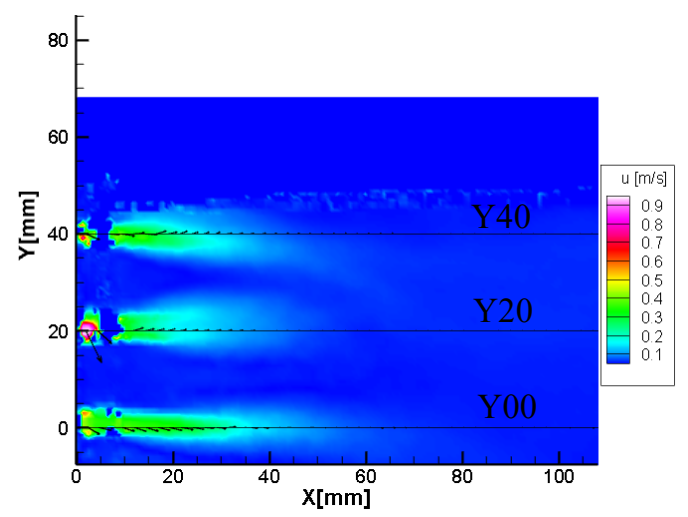

Fig. 7. Velocity fields in longitudinal XY plane according to PIV experimental measurements at $\operatorname{Re} 2500$ in the centre of the jet $(\mathrm{Z}=0 \mathrm{~mm})$ (according to [2]).

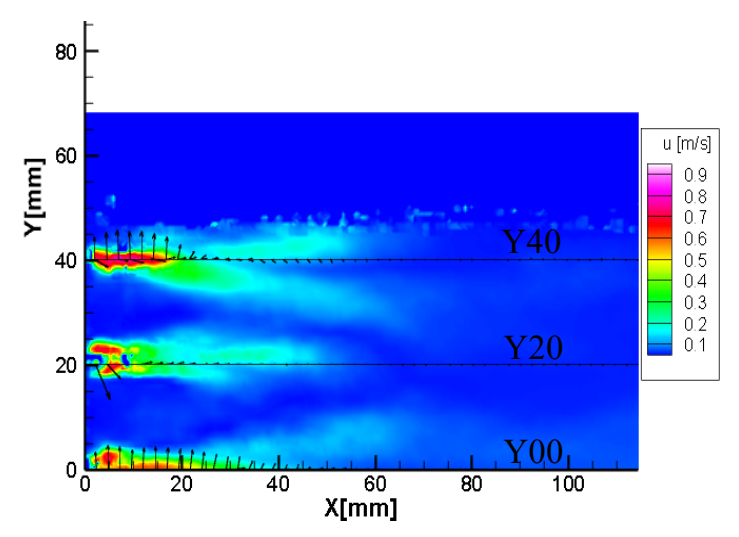

Fig. 8. Velocity fields in longitudinal XY plane according to PIV experimental measurements at Re2500 at $2.5 \mathrm{~mm}$ distance from the jet centre ( $\mathrm{Z}=2.5 \mathrm{~mm})$ (according to [2]).

Figure 7 shows the velocity fields in longitudinal XY plane according to experimental measurements at Re2500 for three different jets (Y00, Y20 and Y40) in their centre $(Z=0 \mathrm{~mm})$. Experimental data was imported and processed in Tecplot for the Y40 jet and the data displayed represents the velocity magnitude. Due to the lack of data in the immediate vicinity of the jet, we decided to process the data starting with $\mathrm{x}=10 \mathrm{~mm}$ and extracted the speed values in 50 points. In the case of the experimental study, the normalized velocity values for the 50 points are shown in Figure 11.

Figure 8 shows the velocity fields in longitudinal XY plane according to experimental measurements at Re2500 for three different jets (Y00, Y20 and Y40) at
$2.5 \mathrm{~mm}$ distance from the jet centre $(Z=2.5 \mathrm{~mm})$. Experimental data was imported and processed in Tecplot for the Y20 jet and the data displayed represents the velocity magnitude. The normalized velocity values for the 50 points are shown in Figure 12.

In the actual case of the solar collector with lobed holes, the air flow rate is $400 \mathrm{~m}^{3} / \mathrm{h}\left(200 \mathrm{~m}^{3} / \mathrm{h} \cdot \mathrm{m}^{2}\right)$, which determines an air velocity in the lobed orifice of 1.312 $\mathrm{m} / \mathrm{s}$ and an air velocity at the computing domain inlet of $0.0555 \mathrm{~m} / \mathrm{s}$, the airflow being characterized by a small Reynolds number $(\mathrm{Re}=422)$. In order to be able to compare the results of the numerical model with the experimental results, the air velocity in the inlet must have a value corresponding to a Reynolds number of $\mathrm{Re}^{\prime}=2500$ in the lobed orifice. To do this, we calculated the new velocity in the lobed orifice $\left(\mathrm{v}_{\mathrm{o}}{ }^{\prime}=7.764 \mathrm{~m} / \mathrm{s}\right)$ and then the resulting velocity at the computing domain inlet $\left(\mathrm{v}_{4 \times 4}{ }^{\prime}=0.375 \mathrm{~m} / \mathrm{s}\right)$, which will be used as inlet boundary condition.

The next step regarding the experimental validation of the numerical model by comparing the velocity profiles in longitudinal planes was the creation of the numerical case. As a result, we imported the 5.3 million cells mesh into ANSYS Fluent and kept the case settings by using the energy conservation equation and k- $\varepsilon$ RNG according to the previous studies conducted [1].

The boundary conditions used are presented in Table 1 , the new air velocity value at the inlet being essential within the validation.

Table 1. Boundary conditions used for the experimental validation.

\begin{tabular}{|c|c|c|}
\hline Boundary condition & Value & Unit \\
\hline $\begin{array}{c}\text { Average velocity at the } \\
\text { computing domain limit (inlet) }\end{array}$ & 0.375 & $\mathrm{~m} / \mathrm{s}$ \\
\hline Ambient temperature (inlet) & 25 & ${ }^{\circ} \mathrm{C}$ \\
\hline Metal plate temperature (metal) & 50 & ${ }^{\circ} \mathrm{C}$ \\
\hline
\end{tabular}

After running the numerical simulation, we obtained the velocity profiles at Re2500 for the airflow through the lobed orifice. The data obtained was imported into the Tecplot data processing software. The XY longitudinal velocity profiles in the centre of the jet and at $2.5 \mathrm{~mm}$ from the centre of the jet are shown in figure 9 and figure 10 . For both $Z=0 \mathrm{~mm}$ and $Z=2.5 \mathrm{~mm}$ cases, we extracted velocity values in 50 points, similar to the previous situations to be able to compare velocity profiles. In the case of the numerical study, normalized values of the velocity magnitude in the 50 points are also presented in figure 11 and figure 12.

In order to properly compare the velocity profiles in both cases, both numerical and experimental results need to be normalized. Figure 11 shows the variation of normalized velocities in 50 points in the longitudinal plane $X Y$ for the plane passing the centre of the jet $(Z=$ $0 \mathrm{~mm})$, both in the case of numerical and experimental study (Y40 jet). It can be observed that the velocity 
profiles are basically the same and after $10 \mathrm{De}$ variation is similar. Figure 12 shows the variation of normalized velocities in 50 points in the longitudinal plane $X Y$ for the plane passing at $2.5 \mathrm{~mm}$ from centre of the jet $(\mathrm{Z}=$ $+2.5 \mathrm{~mm}$ ), both in the case of numerical and experimental study (Y20 jet). It can be observed that the velocity profiles are basically the same and after $10 \mathrm{De}$ variation is similar. The difference between the curves allures is given by the Y20 jet deviation in that zone of the experimental studies.

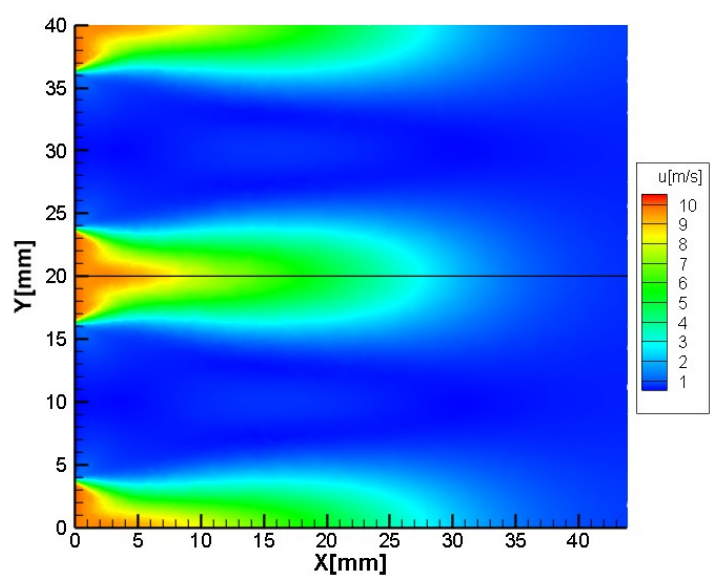

Fig. 9. Velocity field in longitudinal XY plane according to numerical study at $\operatorname{Re} 2500$, in the centre of the jet $(Z=0 \mathrm{~mm})$.

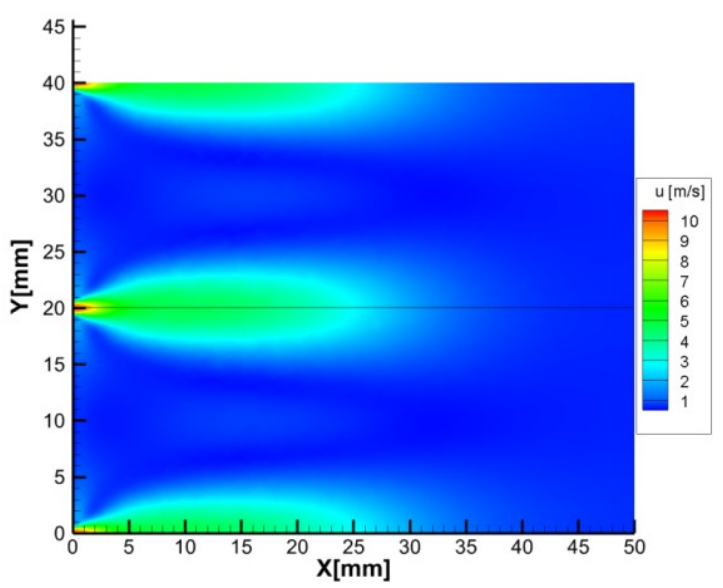

Fig. 10. Velocity field in longitudinal XY plane according to numerical study at Re2500, at $2.5 \mathrm{~mm}$ from the centre of the jet $(\mathrm{Z}=+2.5 \mathrm{~mm})$.

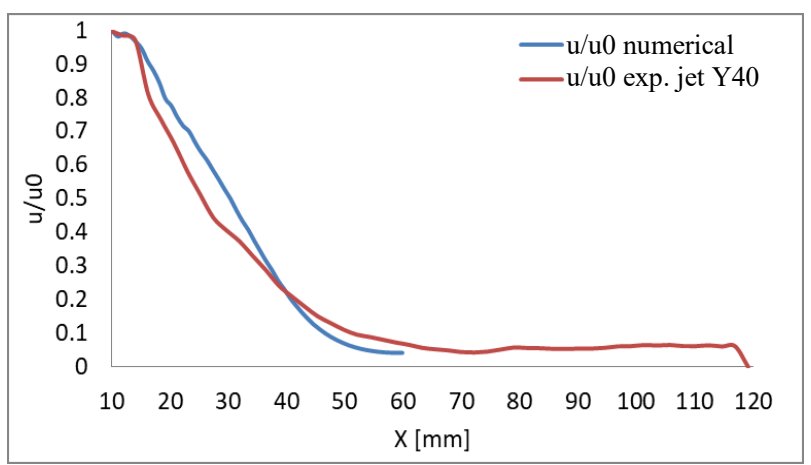

Fig. 11. Normalized velocity magnitude variation: numerical study vs. experimental study (centre of the jet $Z=0 \mathrm{~mm}$ ).

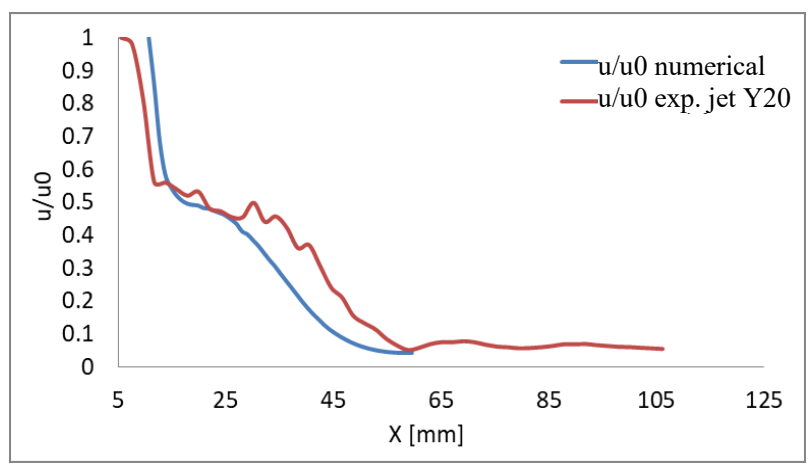

Fig. 12. Normalized velocity magnitude variation: numerical study vs. experimental study (at $2.5 \mathrm{~mm}$ from the centre of the jet $\mathrm{Z}=+2.5 \mathrm{~mm}$ ).

Therefore, by comparing experimental values with numerical results at Re2500, we can conclude that the numerical model reproduces real flow phenomena within acceptable limits. Moreover, starting with 10De velocity profiles are similar, which confirms the premises assumed in the construction of geometry.

\subsection{Outlet temperatures comparison}

Although the experimental validation of the numerical model by comparing velocity profiles in longitudinal plane is the most relevant in the case of the airflow through the absorbent metal plate with lobed holes, we decided to check the temperature value which results on the outlet of the computing domain. In the case of the study of the implementation of phase change materials in solar collectors, the focus is mainly on temperature variations and less on velocities variations, although they can considerably influence the results. The implementation of phase changing materials in transpired solar collectors will be analysed in further studies.

For this validation, we will compare the results in two points of the experimental study (point $1=$ minute 110 and point $2=$ minute 255 ) with the results obtained by running the numerical model in steady state regime. The experimental study was conducted in similar conditions.

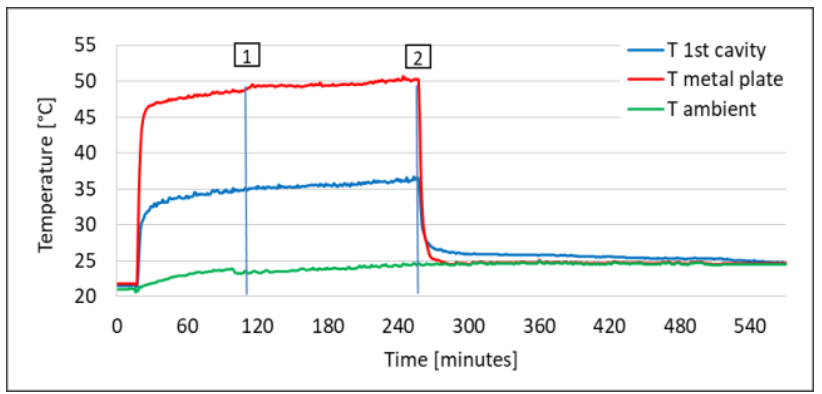

Fig. 13. Temperatures variation in the case of experimental measurements in the two points: 1 and 2 .

As it can be observed in the experimental results presented in the above figure, regarding point 1 (minute 110) at an ambient temperature of $24{ }^{\circ} \mathrm{C}$ and a temperature on the absorbent plate of $49{ }^{\circ} \mathrm{C}$, the air 
temperature in the cavity at $5 \mathrm{~cm}$ distance from the absorbent plate is about $35^{\circ} \mathrm{C}$. Also, regarding point 2 (minute 255) at an ambient temperature of $25^{\circ} \mathrm{C}$ and a temperature on the absorbent plate of $52{ }^{\circ} \mathrm{C}$, the air temperature in the cavity at $5 \mathrm{~cm}$ of the absorbent plate is about $36.5^{\circ} \mathrm{C}$. These were also the boundary conditions used for the numerical study (Table 2 and Table 3).

Table 2. Boundary conditions used for point 1 .

\begin{tabular}{|c|c|c|}
\hline Boundary condition & Value & Unit \\
\hline $\begin{array}{c}\text { Average velocity at the } \\
\text { computing domain limit (inlet) }\end{array}$ & 0.055 & $\mathrm{~m} / \mathrm{s}$ \\
\hline Ambient temperature (inlet) & 24 & ${ }^{\circ} \mathrm{C}$ \\
\hline Metal plate temperature (metal) & 49 & ${ }^{\circ} \mathrm{C}$ \\
\hline
\end{tabular}

Table 3. Boundary conditions used for point 2 .

\begin{tabular}{|c|c|c|}
\hline Boundary condition & Value & Unit \\
\hline $\begin{array}{c}\text { Average velocity at the } \\
\text { computing domain limit (inlet) }\end{array}$ & 0.055 & $\mathrm{~m} / \mathrm{s}$ \\
\hline Ambient temperature (inlet) & 25 & ${ }^{\circ} \mathrm{C}$ \\
\hline Metal plate temperature (metal) & 50 & ${ }^{\circ} \mathrm{C}$ \\
\hline
\end{tabular}

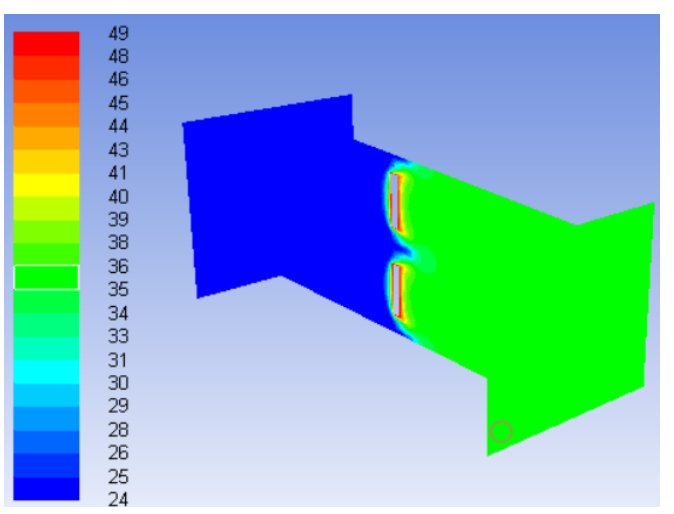

Fig. 14. Temperature fields obtained for the point $1-$ numerical study.

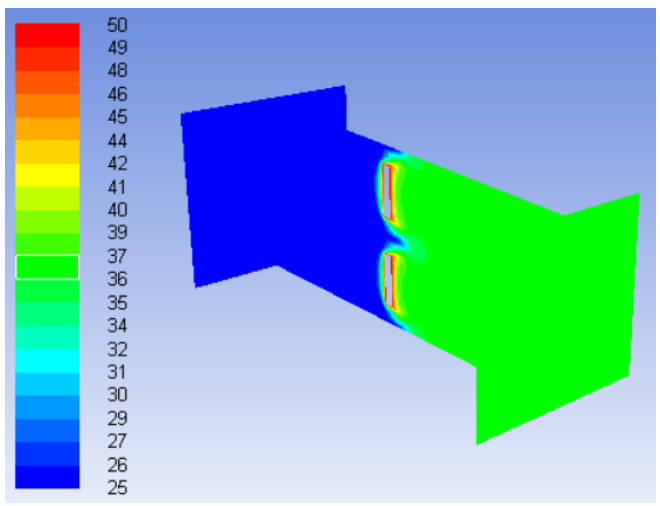

Fig. 15. Temperature fields obtained for the point $2-$ numerical study.
As it can be noticed in figure 14, the temperature field at the outlet, for the 1 st point studied, has a value similar to that obtained in the case of experimental measurements $\left(35^{\circ} \mathrm{C}\right)$. Moreover, the temperatures became uniform quickly after the airflow crosses the absorbent metal plate. Furthermore, as it can be noticed in figure 15 , the temperature field at the outlet, for the 2 nd point studied, has a value similar to that obtained in the case of experimental measurements $\left(36.5^{\circ} \mathrm{C}\right)$.

\section{Final parameters of the numerical model: results and disscusions}

By considering the analyses elaborated in the present paper, we can conclude the final parameters of the numerical model that studies the airflow through the absorbent metal plate with lobed orifices.

According to the mesh independency study conducted in previous studies we chose the 5.3 million cells geometry which will not influence the numerical results, being a stable solution.

We also chose the energy transfer model taking into account the energy conservation equation and the $\mathrm{k}-\varepsilon$ RNG turbulence model (with EWF) which according to the literature is suitable for the type of airflow studied. Having the temperature values at different points from the experimental study, we did not use the heat transfer model for radiation, in order to obtain a simplified model and to facilitate as much as possible the calculations. This model will be developed in the further studies.

For the air circulated in the computing domain we have selected from the ANSYS Fluent software database the appropriate properties, also for the metal absorber plate we have chosen aluminium as the material. The final boundary conditions imposed are those shown in Table 3 , but the values can be modified to obtain various results and to perform parametric studies (postprocessing of the model).

The interpolation scheme used is "second order upwind" for the analysis of convective terms and the pressure-speed coupling scheme is determined by the "SIMPLE" algorithm. According to the literature, the convergence of the solution is achieved when the nondimensional residuals of the flow equations are less than $10^{-3}$; as a result, in order to obtain the relevant results we have imposed a value less than $10^{-5}$.

Below are some of the results of the numerical model built and analysed in this paper, taking into account the boundary conditions shown in Table 3 which capture the "heating" stage of the study.

Figure 17 presents the velocity field at different distances from the absorbent plate: 1De, 5De and 10De ( $5 \mathrm{~cm}$ from the absorbent plate). The last one will be used as boundary condition for the numerical model of the actual solar collector which will be subject of the further studies. Figure 18 presents the velocity and temperature fields in longitudinal planes and figure 19 emphasise the velocity vectors (XY longitudinal plane). Figure 20 presents the velocity vectors for different transverse planes (0De, 2De and 5De) and figure 21-22 highlights the velocity and temperature fields for 7 lobed orifices 
and we can observe that after the metal plate the temperature is rapidly becoming uniform.

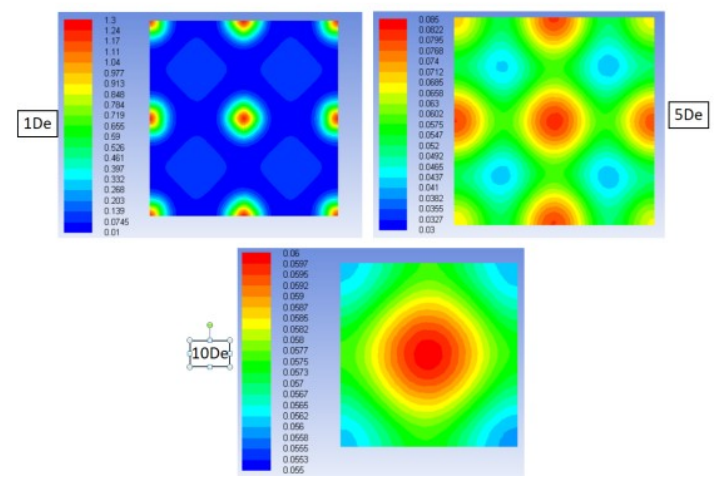

Fig. 17. Velocity fields at different distances from the absorbent plate.

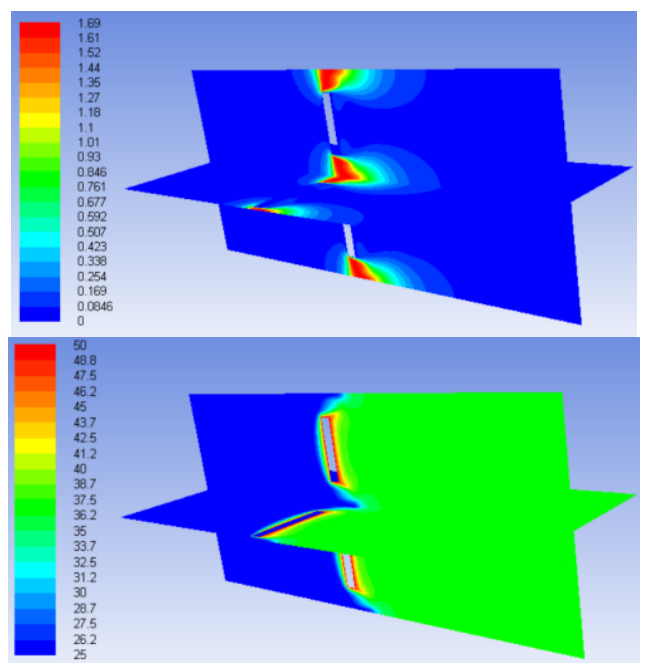

Fig. 18. Velocity and temperature fields in longitudinal $X Y$ and $\mathrm{XZ}$ planes.

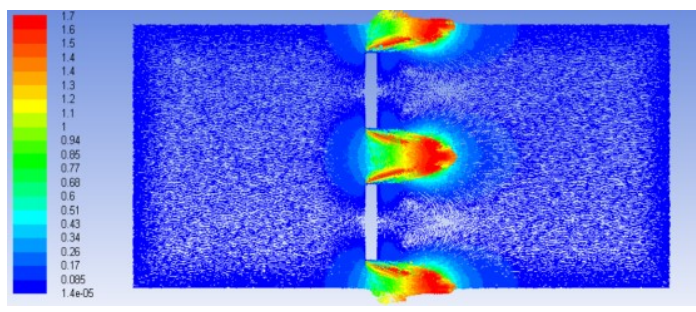

Fig. 19. Velocity vectors in longitudinal XY plane.

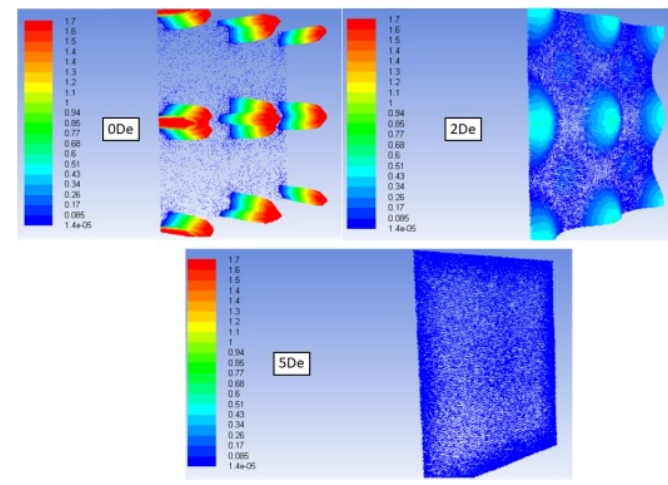

Fig. 20. Velocity vectors in different transverse planes.

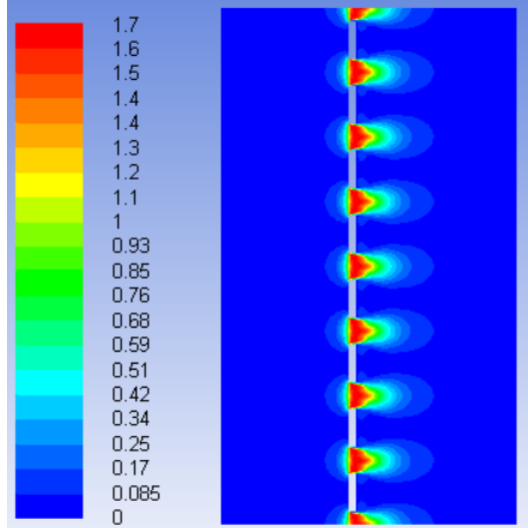

Fig. 21. Velocity fields in longitudinal XY plane for 7 orifices.

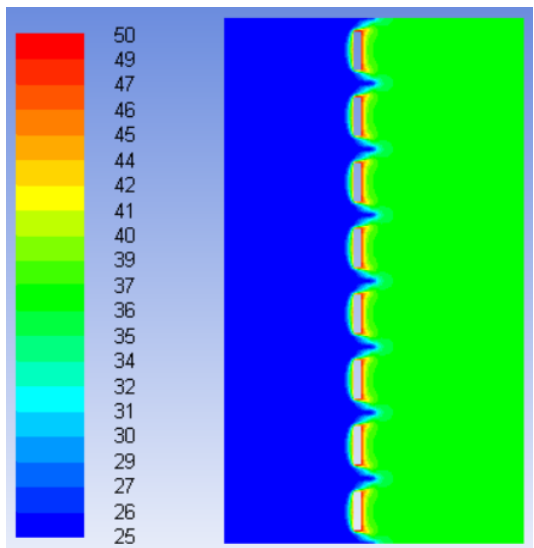

Fig. 22. Temperature fields in longitudinal XY plane for 7 orifices.

\section{Conclusions}

The present paper concludes the final parameters and results of the numerical model that studies the airflow through the lobed orifices of a transpired solar collector which can be used as a solar facade.

By conducting the experimental validation with two different approaches (by comparing velocity profiles in longitudinal planes and by comparing outlet temperature) we can observe that the numerical model reproduces real flow phenomena within acceptable limits.

Obtaining velocity and temperature fields as a result of orifices geometry at 10De will create the opportunity of further parametrical studies. With these results, we can translate into the numerical model of the solar collector the real impact of the lobed geometry. Also, in the future, we will be able to analyse the impact of the orifices on phase change materials implemented in solar collectors. Multiple parametrical studies will be conducted in order to optimise the solar collector geometry and characteristics.

This work was supported by a grant of the Romanian National Authority for Scientific Research, CNCS - UEFISCDI, project number PN-III-P1-1.2-PCCDI-2017-0391. 


\section{References}

1. A.S. Bejan, F. Bode, T. Catalina, and C. Teodosiu, in 1st Conference of the UTCB Doctoral School. 2018.

2. C.V. Croitoru, I. Nastase, F.I. Bode, and A. Meslem, Solar Energy, 131 21-29 (2016).

3. C. Croitoru, I. Nastase, I. Voicu, A. Meslem, and M. Sandu, Energy Procedia, 85 149-155 (2016).

4. L. Navarro, A. de Gracia, D. Niall, A. Castell, M. Browne, S.J. McCormack, P. Griffiths, and L.F. Cabeza, Renewable Energy, 85 1334-1356 (2016).

5. L. Navarro, A. de Gracia, S. Colclough, M. Browne, S.J. McCormack, P. Griffiths, and L.F. Cabeza, Renewable Energy, 88 526-547 (2016).

6. C. Dymond and C. Kutscher, Solar Energy, 60 (5), 291-300 (1997).

7. C. Reichl, K. Kramer, C. Thoma, P. Benovsky, and T. Lemée, Solar Energy, 120 450-463 (2015).

8. B. Molineaux, B. Lachal, and O. Guisan, Solar Energy, 53 (1), 27-32 (1994).

9. L. Gao, H. Bai, and S. Mao, Energy Conversion and Management, 77 690-699 (2014).

10. G.W.E. Van Decker, K.G.T. Hollands, and A.P. Brunger, Solar Energy, 71 (1), 33-45 (2001).

11. Amina Meslem, Ilinca Nastase, Florin Bode, and Claudine Beghein, The International Journal of Ventilation, 11 (3), 16 (2012).

12. Amina Meslem, Florin Bode, Ilinca Nastase, and Olivier Martin, Modern Applied Science, 6 (12), 15 (2012).

13. Florin Bode, Ilinca Nastase, and Cristiana Croitoru, Mathematical Modelling in Civil Engineering - Scientific Journal, 7 (4), 7 (2011).

14. Florin Bode, Kodjovi Sodjavi, Amina Meslem, and I. Nastase, SCIENTIFIC BULLETIN University Politehnica of Bucharest, Series D: Mechanical Engineering, 76 (12), 8 (2014).

15. E. Osterman, V. Butala, and U. Stritih, Energy and Buildings, 106 125-133 (2015).

16. M. Gholampour and M. Ameri, Appl. Energy 164, (2016).

17. M. Badache, D. Rousse, S. Halle, G. Quesada, and Y. Dutil, Energy Procedia, (2012).

18. ANSYS, Inc "Fluent 19.0 User Guide".

19. A.S. Bejan, M. Sandu, L. Tacutu, C. Croitoru, and I. Nastase. in Roomvent \& Ventilation 2018. 2018. Finland.

20. Manual:. ANSYS Fluent Chapter 12.4.2 RNG kepsilon Model. accessed march 2018; Available from:

www.sharcnet.ca/Software/Fluent6/html/ug/nod e479.htm.

21. D. Bordianu, C. Croitoru, and I. Nastase, Master Thesis, (2016). 